Supplementary Material for:

\title{
Development of a Wet Chemical Method for the Speciation of Iron in Atmospheric Aerosols
}

\author{
Brian J. Majestic, James J. Schauer ${ }^{*}$, Martin M. Shafer \\ Environmental Chemistry and Technology Program \\ University of Wisconsin - Madison \\ 660 N. Park St \\ Madison, WI 53706 \\ Jay Turner \\ Environmental Engineering Program \\ Washington University of St. Louis \\ One Brookings Drive Campus Box 1180 \\ St. Louis, MO 63130 \\ Philip M. Fine, Manisha Singh, Constantinos Sioutas \\ Department of Civil and Environmental Engineering \\ University of Southern California \\ 3620 South Vermont Avenue \\ Los Angeles, CA 90089
}


Coal fly ash was collected from the stoker-boiler coal-fired power plant in. The fly ash was then passed through a PM10 cyclone and resuspended on $47 \mathrm{~mm}$ Teflo (Pall Life Sciences, $2.0 \mu \mathrm{m}$ ) filters. Duplicate filters resuspended fly ash was then analyzed by ICP-MS for total metals and the Ferrozine method for iron oxidation state specific determination (the $\mathrm{NaCl}$ extractant was not measured). The results (on the bases of total fly ash and iron in the fly ash) are shown in the figure below. As the figure shows, labile Fe(II) ranged from $0.001 \%$ (MQ) to $0.003 \%$ (Acetate) of the total fly and $0.09 \%$ up to $0.17 \%$ of the total iron pool.

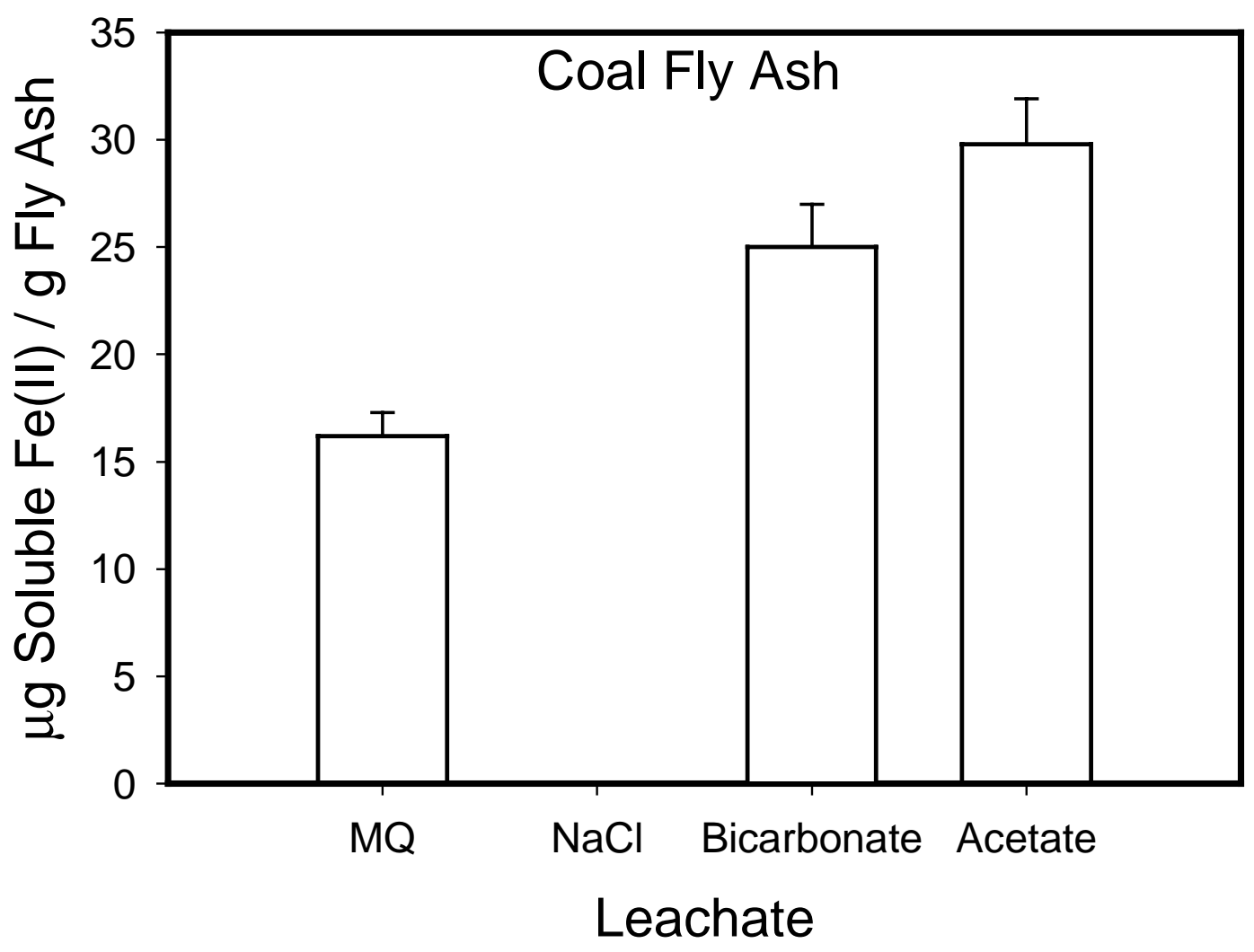




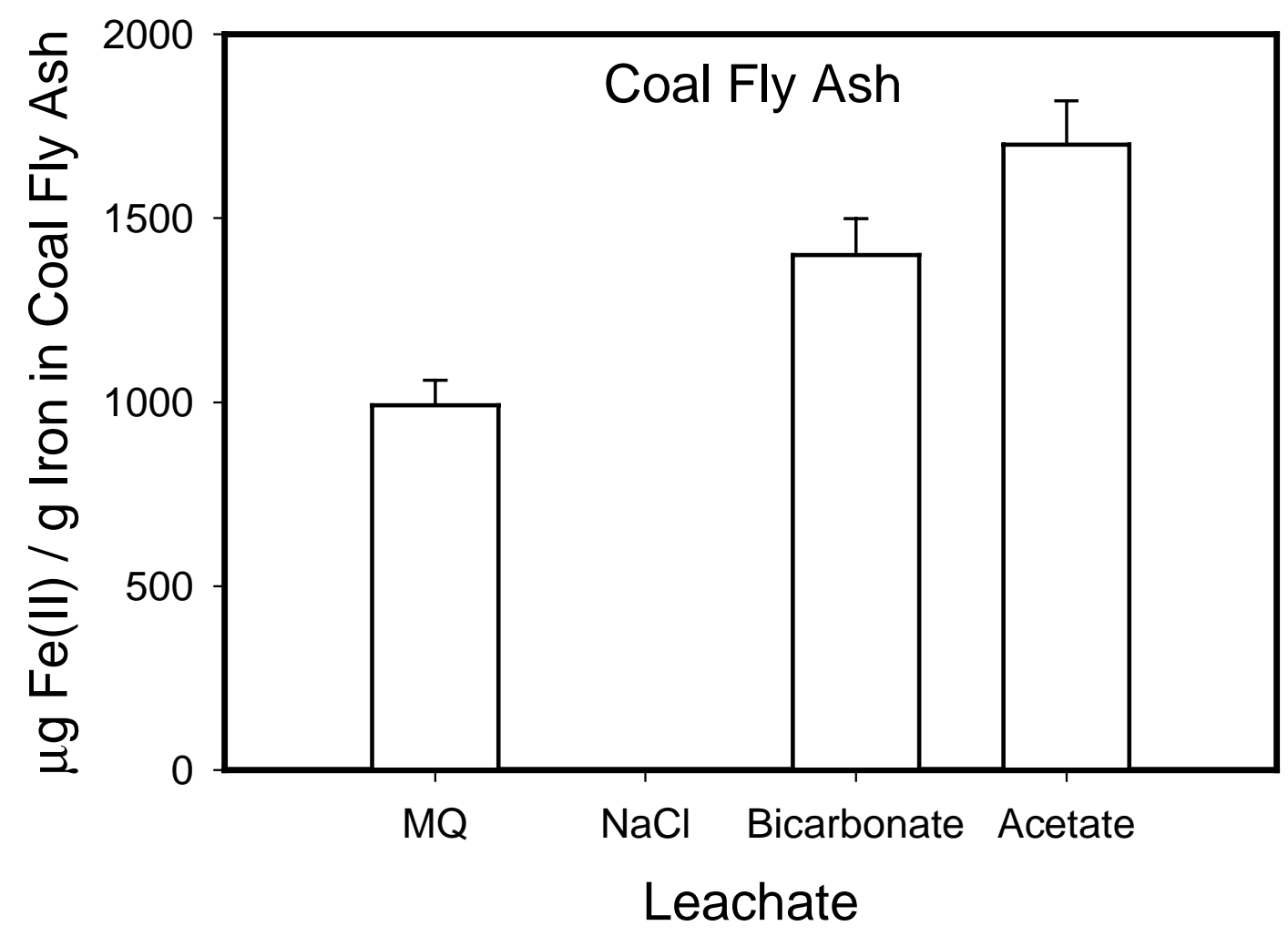

